\title{
Ultraviolet photoactivation using Synchrotron Radiation for Tandem Mass Spectrometry of Polysiloxanes
}

\author{
Inès Aloui ${ }^{1}$, Véronique Legros ${ }^{2}$, Alexandre Giuliani ${ }^{3,4}$, William Buchmann ${ }^{1 *}$ \\ *william.buchmann@univ-evry.fr \\ ${ }^{1}$ Université Paris-Saclay, Univ Evry, CNRS, LAMBE, 91025, Evry-Courcouronnes, France. \\ ${ }^{2}$ Proteomic Platform, Institut Jacques Monod, 75205 Paris 13, France. \\ ${ }^{3}$ Disco Beamline, Université Paris-Saclay, Synchrotron Soleil, 91190, Saint-Aubin, France. \\ ${ }^{4}$ UAR1008, Transform, INRAe, Rue de la Géraudière, F-44316 Nantes, France.
}

\section{Supporting information}


Figure S1 shows the product ion mass spectrum obtained from $\left[\mathrm{PDMS}_{25}+\mathrm{Na}\right]^{+}$at $\mathrm{m} / \mathrm{z} 1961.5$ by collisional activation with helium. No fragmentation was observed from $\left[\mathrm{PDMS}_{25}+\mathrm{Na}\right]^{+}$ under CID conditions.

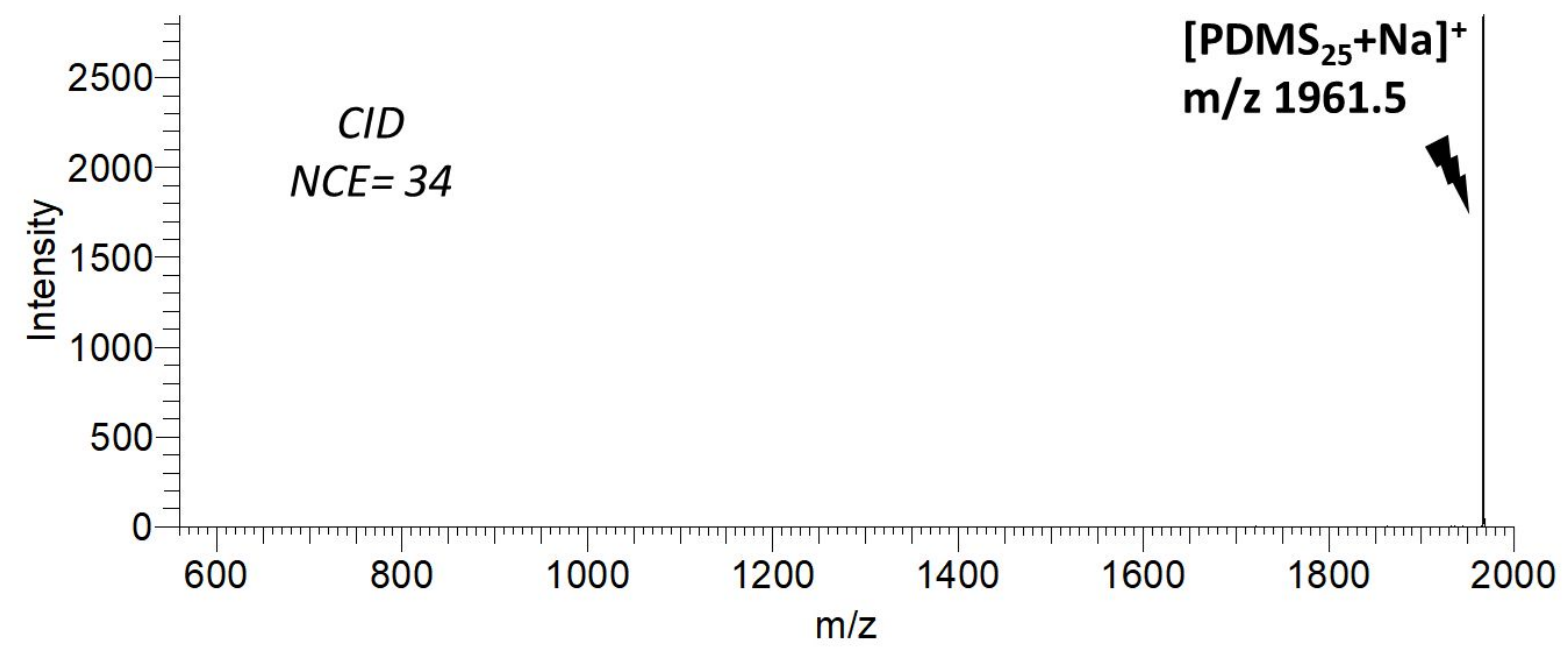

Figure S1. CID mass spectrum obtained from $\left[\mathrm{PDMS}_{25}+\mathrm{Na}\right]^{+}$at $\mathrm{m} / \mathrm{z} 1961.5$ (normalized collision energy (NCE): 34, number of scans: 100).

The electron transfer dissociation (ETD) experiment was performed using a hybrid linear ion trap Orbitrap XL ETD mass spectrometer (Thermo Fisher Scientific) equipped with an electrospray ionization (ESI) source. Fluoranthene radical anions were generated in a negative ionization source, which were transported to the ion trap and used as the electron-transfer agent. ETD mass spectrum obtained from the precursor ion $\left[\mathrm{PDMS}_{25}+2 \mathrm{Na}\right]^{2+}$ at $\mathrm{m} / \mathrm{z} 992.3$. The doubly charged ion $\left[\mathrm{PDMS}_{25}+2 \mathrm{Na}\right]^{2+}$ was involved rather than the singly charged ion $\left[\mathrm{PDMS}_{25}+\mathrm{Na}\right]^{+}$ because this latter cannot be activated under ETD conditions, the electron transfer on a singly charged precursor ion resulting in a neutral specie. 


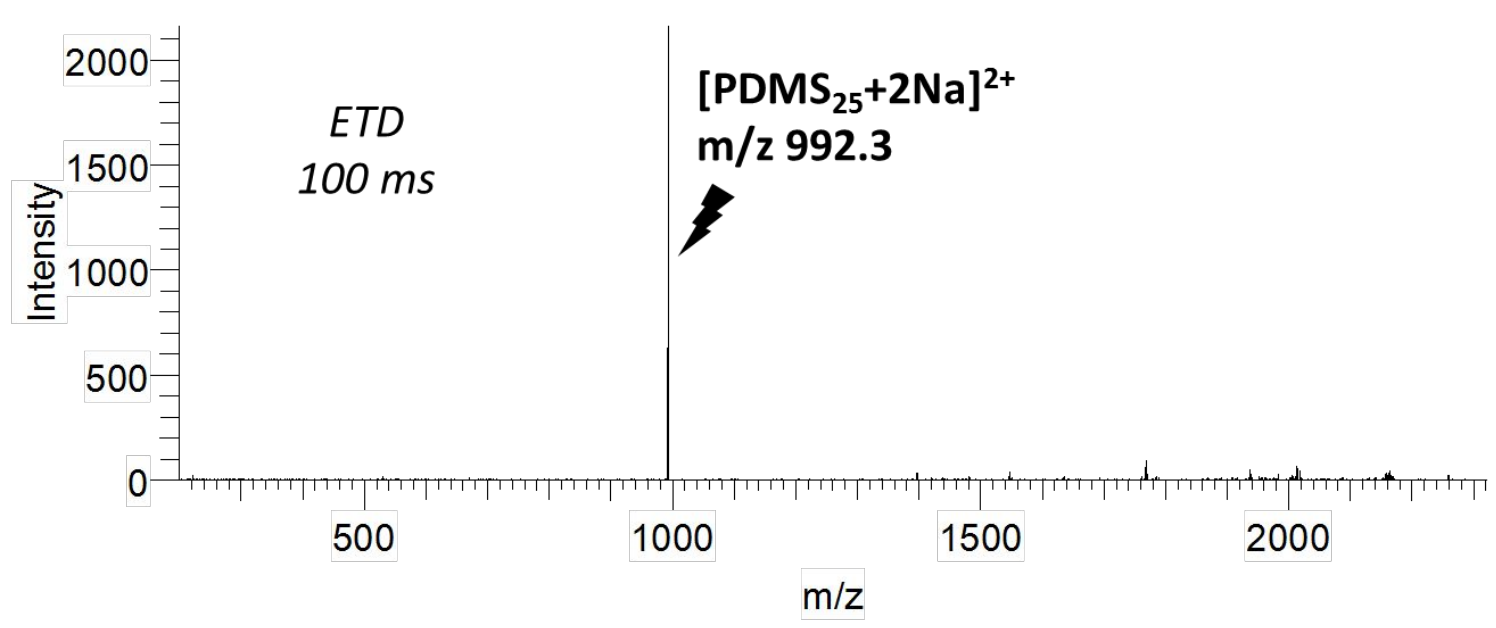

Figure S2. ETD mass spectrum obtained from $\left[\mathrm{PDMS}_{25}+2 \mathrm{Na}\right]^{2+}$ at $\mathrm{m} / \mathrm{z} 992.3$ (activation time: $100 \mathrm{~ms}$, number of scans: 100).

Figure S3 displays the product ion mass spectrum obtained from $\left[\mathrm{PDMS}_{25}+\mathrm{Na}\right]^{+}$ion at $\mathrm{m} / \mathrm{z}$ 1961.5 followed by synchrotron UV irradiation at $20 \mathrm{eV}$ photon energy during $100 \mathrm{~ms}$. The parameter $\mathrm{Q}$ was set to 0.025 . One radical dication is observed at $\mathrm{m} / \mathrm{z} 973.3$, along with two different ion series labelled $a_{n}{ }^{2+}$ and $b_{n}$, and few fragment ions labelled $a_{n}{ }^{+}$corresponding to the $\mathrm{a}_{\mathrm{n}}{ }^{2+}$ series without the $\mathrm{Na}^{+}$.

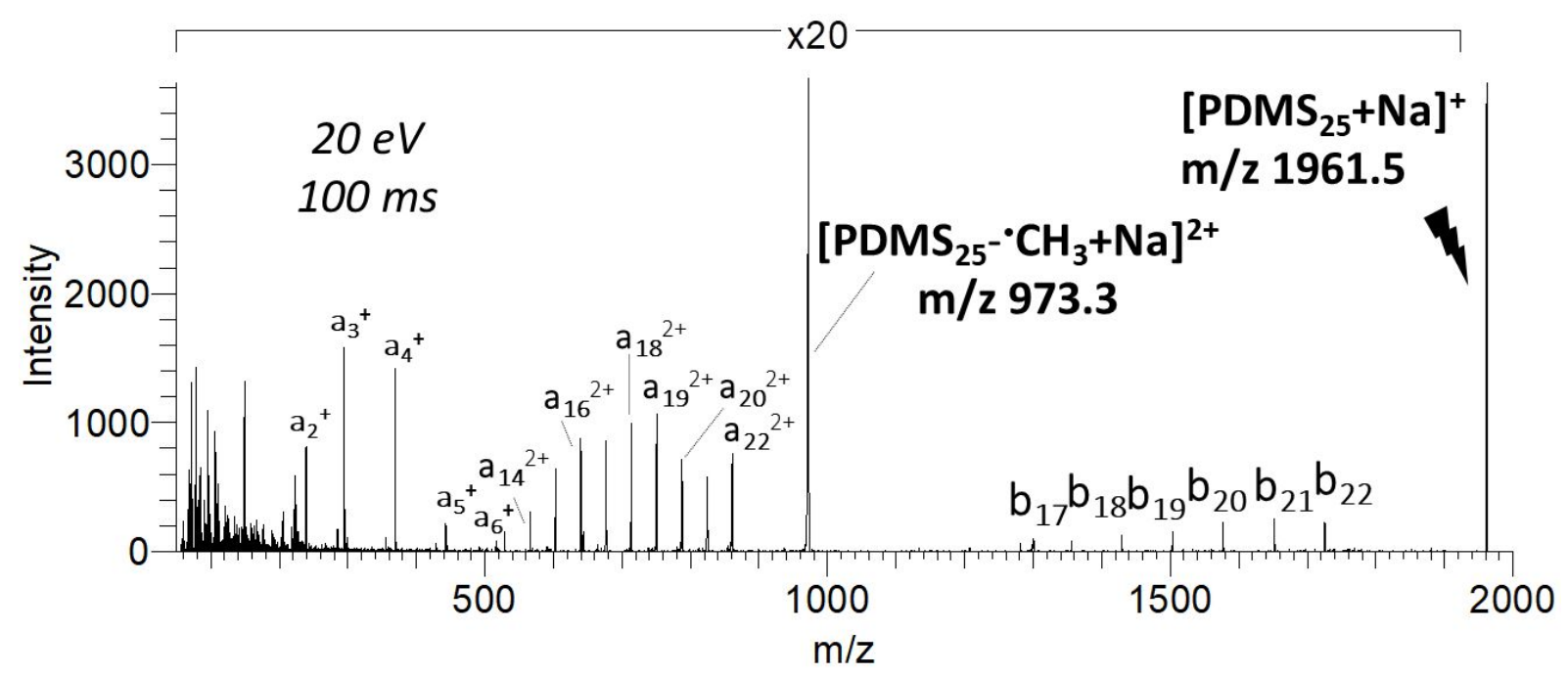

Figure S3. Ultraviolet-photoactivation mass spectra obtained following UV activation of $\left.{ }_{\mathrm{PDMS}}{ }_{25}+\mathrm{Na}\right]^{+}$ion at $\mathrm{m} / \mathrm{z} 1961.5$ (Photon energy : $20 \mathrm{eV}$, irradiation time: $100 \mathrm{~ms}$, number of scans: 100).

After the isolation of $\left[\mathrm{PDMS}_{25^{-}}{ }^{-} \mathrm{CH}_{3}+\mathrm{Na}\right]^{2+}$ ion at $\mathrm{m} / \mathrm{z} 973.3$ during $300 \mathrm{~ms}$, initially produced by $\mathrm{UV}$ activation at $20 \mathrm{eV}(5000 \mathrm{~ms}), \mathrm{a}_{\mathrm{n}}{ }^{2+}, \mathrm{b}_{\mathrm{n}}, \mathrm{b}_{\mathrm{n}}{ }^{\prime \prime}$ and $\mathrm{a}_{\mathrm{n}}{ }^{+}$ion series were produced (Figure S4). 


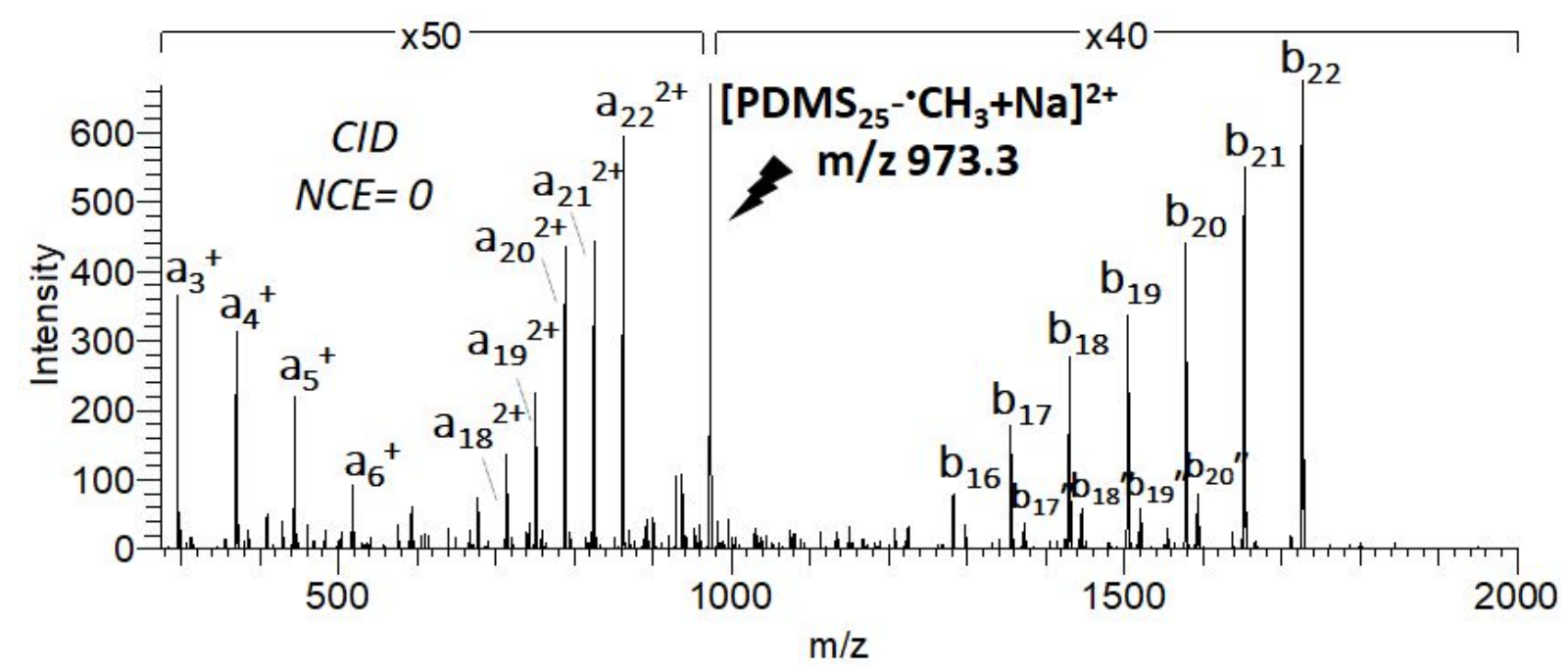

Figure S4. Product ion mass spectrum of $\left[\mathrm{PDMS}_{25^{-}}{ }^{\circ} \mathrm{CH}_{3}+\mathrm{Na}\right]^{2+}$ at $\mathrm{m} / \mathrm{z} 973.3$, initially produced by $\mathrm{UV}$ activation at $20 \mathrm{eV}(5000 \mathrm{~ms})$ from $\left[\mathrm{PDMS}_{25}+\mathrm{Na}\right]^{+}$, obtained after the isolation of $\left[\mathrm{PDMS}_{25} \cdot{ }^{\cdot} \mathrm{CH}_{3}+\mathrm{Na}\right]^{2+}$ in the linear ion trap during $300 \mathrm{~ms}$ (NCE: 0 ). 